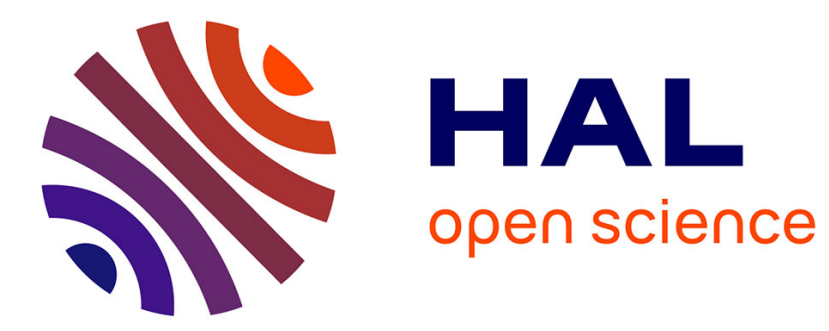

\title{
L1-optimal linear programming estimator for periodic frontier functions with Hölder continuous derivative
}

Alexander Nazin, Stéphane Girard

\section{To cite this version:}

Alexander Nazin, Stéphane Girard. L1-optimal linear programming estimator for periodic frontier functions with Hölder continuous derivative. Automation and Remote Control / Avtomatika i Telemekhanika, 2014, 75 (12), pp.2152-2169. 10.1134/S0005117914120066 . hal-01066739

\section{HAL Id: hal-01066739 \\ https://hal.science/hal-01066739}

Submitted on 22 Sep 2014

HAL is a multi-disciplinary open access archive for the deposit and dissemination of scientific research documents, whether they are published or not. The documents may come from teaching and research institutions in France or abroad, or from public or private research centers.
L'archive ouverte pluridisciplinaire HAL, est destinée au dépôt et à la diffusion de documents scientifiques de niveau recherche, publiés ou non, émanant des établissements d'enseignement et de recherche français ou étrangers, des laboratoires publics ou privés. 


\title{
$L_{1}$-optimal linear programming estimator for periodic frontier functions with Hölder continuous derivative
}

\author{
A.V. Nazin.* \\ (Trapeznikov Institute of Control Sciences RAS, Moscow, Russia), \\ S. Girard. \\ (LJK, Inria Grenoble Rhône-Alpes, Grenoble, France)
}

\begin{abstract}
We propose a new estimator based on a linear programming method for smooth frontiers of sample points. The derivative of the frontier function is supposed to be Hölder continuous. The estimator is defined as a linear combination of kernel functions being sufficiently regular, covering all the points and whose associated support is of smallest surface. The coefficients of the linear combination are computed by solving a linear programming problem. The $L_{1}$ error between the estimated and the true frontier functions is shown to be almost surely converging to zero, and the rate of convergence is proved to be optimal.
\end{abstract}

\section{Introduction}

Many proposals are given in the literature for estimating a set $S$ given a finite random set of points drawn from the interior. Here, we focus on the case where the unknown support can be written as $S=\{(x, y): 0 \leq x \leq 1 ; 0 \leq y \leq f(x)\}$, where $f$ is an unknown function. The initial problem reduces to estimating $f$, called the frontier or the boundary, from random pairs $(X, Y)$ included in $S$.

Under monotonicity assumptions, the frontier can also be interpreted as the endpoint of $Y$ given $X \leq x$. Specific estimation techniques have been developed in this context, see for instance Deprins et al. [6], Farrel [7], Gijbels et al. [9]. We also refer to Aragon et al. [1], Cazals et al. [4], DaOuia \& Simar [5] for the definition of robust estimators.

In the general case, that is without monotonicity assumptions, GIRARD \& JACOB [16] introduced an estimator based upon kernel regression on high power-transformed data. In the particular case where $Y$ given $X=x$ is uniformly distributed they proved that this estimator is asymptotically Gaussian with the minimax rate of convergence for Lipschitzian frontiers. (Loosely speaking, under the rate of convergence we understand infinitely small positive number sequence which characterizes the convergence to zero of a norm of the estimation error, as the sample size $N \rightarrow \infty$.) Compared to the extreme-value based estimators

\footnotetext{
${ }^{*}$ The work of A.V. Nazin was carried out during his stay in MISTIS Project, Inria Grenoble Rhône-Alpes, June and October 2013; partially supported by PreMoLab/MIPT, RF government grant 11.G34.31.0073.
} 
(Geffroy [8], Girard \& Jacob [13, 14, 15], Girard \& Menneteau [17], Härdle et al. [20], Menneteau [25]), projection estimators (Jacob \& Suquet [21]), or piecewise polynomial estimators (Hall et al. [18], Knight [22], Korostelev \& Tsybakov [24], Korostelev et al. [23], HÄrdle et al. [19]), this estimator does not require a partition of the support $S$. When the conditional distribution of $Y$ given $X$ is not uniform, this estimator is still convergent (GIRARD \& JACOB [16], Theorem 1) but may suffer from a strong bias (GIRARD \& JACOB [16], Table 1). A modification of this estimator has been proposed by GIRARD et al. $[10,11]$ to tackle the situation where the conditional distribution function of $Y$ given $X=x$ decreases at a polynomial rate to zero in the neighborhood of the frontier $f(x)$. The asymptotic normality as well as the strong consistency of the estimator are established.

The estimator proposed in BOUCHARD et al [2] for estimating $S$ shares some common characteristics with the one of Girard \& JACOB [16]. It assumes that $Y$ given $X=x$ is uniformly distributed but does not require a partition of the support. Besides, it is defined as a kernel estimator obtained by smoothing some selected points of the sample. These points are, however, chosen automatically by solving a linear programming problem to obtain an estimated support covering all the points and with smallest surface. From the theoretical point of view, this estimator is shown to be consistent for the $L_{1}$ norm. An improvement of this estimator has been proposed in GIRARD et al. [12] in order to reach the optimal minimax $L_{1}$ rate of convergence (up to a logarithmic factor) for Lipschitzian frontiers.

In this paper, we propose an adaptation of these methods for estimating smoother frontiers: It is assumed that the first derivative of frontier is Hölder continuous. The resulting estimator is proved to reach the optimal minimax $L_{1}$ rate of convergence (up to a logarithmic factor). The paper is organized as follows. The estimator is defined in Section 2. Assumptions and preliminary results are given in Section 3 while our main result is established in Section 4. Proofs are postponed to the Appendix.

\section{Problem statement and boundary estimator}

Let all the random variables be defined on a probability space $(\Omega, \mathcal{F}, P)$. The problem under consideration is to estimate an unknown 1-periodic function $f: \mathbb{R} \rightarrow(0, \infty)$, that is $f(x+1)=f(x)$ for all $x \in \mathbb{R}$, on the basis of independent observations $\left(X_{i}, Y_{i}\right)_{i=\overline{1, N}}$ uniformly distributed in

$$
S \triangleq\{(x, y): 0 \leq x \leq 1,0 \leq y \leq f(x)\}
$$

Note that, the notation $i=\overline{m, n}$ is used for $i=m, \ldots, n$. Since $f$ is 1-periodic, it is convenient to extend the indices of data $\left(X_{i}, Y_{i}\right)$ out of those of $i=\overline{1, N}$ by periodic continuation w.r.t. $x$. Therefore, we put $\left(X_{i}, Y_{i}\right)=\left(X_{i+N}-1, Y_{i+N}\right)$ for $i=\overline{1-N, 0}$, and $\left(X_{i}, Y_{i}\right)=\left(X_{i-N}+1, Y_{i-N}\right)$ for $i=\overline{N+1,2 N}$.

Remark 1 An example of the boundary of $2 \pi$-periodic function occurs in the description of the boundary of a convex planar set in polar coordinates with the center inside the set. The normalization of the polar angle allows to come to 1-periodic function. 
Remark 2 Note that the condition of periodicity of the boundary function is, on the one hand, a significant assumption that distinguish a special class of problems, on the other hand, the technical condition that allows (together with the conditions on the kernel function, see below) to avoid the "difficulties at the borders" of the interval $[0,1]$, simplify the calculations in the analysis of the estimation error and arrive to the optimal rate of convergence.

Letting

$$
C_{f} \triangleq \int_{0}^{1} f(u) d u
$$

each variable $X_{i}$ is distributed in $[0,1]$ with p.d.f. $f(\cdot) / C_{f}$ while $Y_{i}$ has an uniform conditional distribution with respect to $X_{i}$ in the interval $\left[0, f\left(X_{i}\right)\right]$. In what follows, it is assumed that $f \in \Sigma\left(1, \beta, L_{\beta}\right), 1<\beta \leq 2$, i.e. the function $f: \mathbb{R} \rightarrow(0, \infty)$ is 1-periodic, continuously differentiable with Hölder continuous derivative $f^{\prime}$ having exponent $\beta-1$ and upper bound for Hölder coefficient $L_{\beta}$ :

$$
\left|f^{\prime}(x)-f^{\prime}(u)\right| \leq L_{\beta}|x-u|^{\beta-1} \quad \forall x, u \in \mathbb{R} .
$$

The considered estimator $\widehat{f}_{N}:[0,1] \rightarrow[0, \infty)$ of the frontier is chosen from the family of functions

$$
\widehat{f}_{N}(x)=\sum_{i=1}^{N} \alpha_{i} K_{h}\left(x, X_{i}\right), \quad \alpha_{i} \geq 0, \quad i=1, \ldots, N
$$

with kernel function

$$
K_{h}(x, t)=\frac{1}{h} K\left(\frac{x-t}{h}\right)+\mid \begin{array}{ll}
0 & \text { if } h<t<1-h, \\
\frac{1}{h} K\left(\frac{x-t-1}{h}\right) & \text { if } 0 \leq t \leq h, \\
\frac{1}{h} K\left(\frac{x-t+1}{h}\right) & \text { if } 1-h \leq t \leq 1,
\end{array}
$$

being defined for all $(x, t) \in[0,1]^{2}$ and $h \in(0,1 / 2)$, where $K$ is a given sufficiently smooth centered density function $K: \mathbb{R} \rightarrow[0, \infty)$ with support included in $[-1,1]$, see assumption B2, Section 3. The bandwidth parameter $h$ depends on $N$ such that $h \rightarrow 0$ as $N \rightarrow \infty$.

Remark 3 The estimate (4) is not supposed to be periodic itself.

Remark 4 Since the supports of the different terms appearing in (5) do not intersect, the kernel may be rewritten as

$$
K_{h}(x, t)=\sum_{j=-1}^{1} \frac{1}{h} K\left(\frac{x-t+j}{h}\right)
$$

for $(x, t) \in[0,1]^{2}$. The kernel function (6) is thus as smooth w.r.t. $t$ as the density function $K(\cdot)$ is. For instance, one always has $K_{h}(x, t) \leq K_{\max } / h$ and the $k$-th derivative bound $\left|\partial^{k} K_{h}(x, t) / \partial t^{k}\right| \leq K_{\max }^{(k)} / h^{k+1}$ if $K(\cdot)$ has a continuous $k$-th derivative. 
Remark 5 The optimal choice of the bandwidth $h$ is carried out here in the spirit of [12]. Also see Remark 7 below at the end of Section 6.3.

Remark 6 In the definition of estimator (4), the kernel (5) is introduced for $x \in[0,1]$. However, it is convenient to introduce a wider interval like $[-h, 1+h]$ for variables $x$ and $t$ in the kernel function (5) and define additional points $X_{-} \in[-h, 0]$ and $X_{+} \in[1,1+h]$ a.s., see below (20).

As it is proved below in Lemma 2 the surface of the estimated support

$$
\widehat{S}_{N} \triangleq\left\{(x, y): 0 \leq x \leq 1,0 \leq y \leq \widehat{f}_{N}(x)\right\}
$$

is given by

$$
\int_{0}^{1} \widehat{f}_{N}(x) d x=\sum_{i=1}^{N} \alpha_{i} .
$$

This suggests to define the estimator of the parameter vector $\alpha=\left(\alpha_{1}, \ldots, \alpha_{N}\right)^{T}$ as a solution of the following optimization problem

$$
J_{P}^{*} \triangleq \min _{\alpha} \sum_{i=1}^{N} \alpha_{i}
$$

subject to,

$$
\begin{aligned}
& \text { for all } i=\overline{1, N}, \\
& \widehat{f}_{N}\left(X_{i}\right)+\left(X_{j}-X_{i}\right) \widehat{f}_{N}^{\prime}\left(X_{i}\right) \geq Y_{j}, \quad \forall j:\left|X_{j}-X_{i}\right| \leq h, \\
& \left|\widehat{f}_{N}^{\prime \prime}\left(X_{i}\right)\right| \leq 2 L_{\beta} K_{\max }^{\prime \prime} \frac{\log N}{N h^{3}}, \\
& \sum_{i=1}^{N} \alpha_{i} 1\left\{(m-1) / m_{h} \leq X_{i}<m / m_{h}\right\} \leq C_{\alpha} h, \quad m=\overline{1, m_{h}}, \\
& 0 \leq \alpha_{i},
\end{aligned}
$$

where $m_{h}=\lfloor 1 / h\rfloor$ is the integer part of $1 / h$,

$$
K^{\prime \prime}(x, u) \triangleq \frac{\partial^{2}}{\partial x^{2}} K_{h}(x, u), \quad(x, u) \in[0,1]^{2},
$$

and $\mathbf{1}\{\cdot\}$ is the indicator function which equals 1 if the argument condition holds true, and 0 otherwise. The value of the positive parameter $C_{\alpha}$ in the constraints (12) is discussed in Section 4. Evidently, this optimization problem represents a linear program (LP). Therefore, we call the defined boundary estimator as the LP-estimator (9)-(13).

\section{Basic assumptions and preliminary results}

The basic assumptions on the unknown boundary function $f: \mathbb{R} \rightarrow(0,+\infty)$ are:

A1. $f(x)=f(x+1)$ and $0<f_{\min } \leq f(x) \leq f_{\max }<\infty$, for all $x \in \mathbb{R}$. 
A2. $f(x)$ is continuously differentiable having the Hölder exponent $\beta-1$ for function derivative $f^{\prime}$, i.e.

$$
\left|f^{\prime}(x)-f^{\prime}(y)\right| \leq L_{\beta}|x-y|^{\beta-1} \quad \text { for all } \quad x, y \in[0,1]
$$

where constants $L_{\beta}<\infty$ and $\beta \in(1,2]$ are supposed to be given.

The following assumptions on the kernel function are introduced:

B1. $K: \mathbb{R} \rightarrow[0,+\infty)$ has a compact support: $\operatorname{supp}_{t \in \mathbb{R}} K(t)=[-1,1]$,

B2. $\int_{-1}^{1} K(t) d t=1, \int_{-1}^{1} t K(t) d t=0$

B3. $K$ is four times continuously differentiable.

The next two lemmas are of analytical nature. They can be interpreted as extensions of Bochner's Lemma for controlling the smoothing error introduced by the kernel.

Lemma 1 Let $f$ be a 1-periodic function on $\mathbb{R}$. Then, assumptions B1 and B2 imply

$$
\int_{0}^{1} f(u) K_{h}(x, u) d u=\int_{-1}^{1} f(x-h v) K(v) d v \quad \forall x \in[0,1] .
$$

Particularly, for $f(x) \equiv 1$ one obtain

$$
\int_{0}^{1} K_{h}(x, u) d u=1 \quad \forall x \in[0,1] .
$$

In addition,

$$
\begin{aligned}
\int_{0}^{1}(u-x) K_{h}(x, u) d u & =0 & \forall x \in[h, 1-h], \\
\int_{0}^{1} K_{h}(x, u) d x & =1 & \forall u \in[0,1], \\
\int_{0}^{1}(x-u) K_{h}(x, u) d x & =0 & \forall u \in[0,1] .
\end{aligned}
$$

Now, we quote several preliminary results on the estimator $\widehat{f}_{N}$. First, Lemma 2 establishes that the surface of the related estimated support $\widehat{S}_{N}$ equals $\sum_{i=1}^{N} \alpha_{i}$. Second, functions $\widehat{f}_{N}$ and $\widehat{f}_{N}^{\prime}$ are proved to be Lipschitzian, see Lemma 3 and Lemma 4 respectively. Proofs are postponed to Subsection 6.1.

Lemma 2 Suppose B1, B2 are verified and $0<h<1 / 2$. Then, the surface of the estimated support (7) is

$$
\int_{0}^{1} \widehat{f}_{N}(x) d x=\sum_{i=1}^{N} \alpha_{i}
$$


Introduce additionally

$$
X_{-} \triangleq \max _{i=1, N}\left\{X_{i}\right\}-1, \quad X_{+} \triangleq \min _{i=1, N}\left\{X_{i}\right\}+1 .
$$

Lemma 3 Suppose A1, A2, and B1, B3 are verified. Then, the Lipschitz constant of the LP-estimator (9)-(13) is bounded by

$$
L_{\widehat{f}_{N}} \triangleq \max _{x \in\left[X_{-}, X_{+}\right]}\left|\widehat{f}_{N}^{\prime}(x)\right| \leq 3 C_{\alpha} K_{\max }^{\prime} h^{-1} .
$$

Lemma 4 Suppose A1, A2, and B1, B3 are verified. Moreover, let $h \rightarrow 0$ as $N \rightarrow \infty$ such that

$$
\lim _{N \rightarrow \infty} \frac{\log N}{N h}=0
$$

Then, there exists almost surely finite $N_{4}=N_{4}(\omega)$ such that for any $N \geq N_{4}$ the Lipschitz constant for the derivative estimator $\widehat{f}_{N}^{\prime}$ over interval $\left[X_{-}, X_{+}\right] \supseteq[0,1]$ is bounded as follows:

$$
L_{\widehat{f}_{N}^{\prime}} \triangleq \max _{x \in\left[X_{-}, X_{+}\right]}\left|\widehat{f}_{N}^{\prime \prime}(x)\right| \leq 4 L_{\beta} K_{\max }^{\prime \prime} \frac{\log N}{N h^{3}} .
$$

Below in the next section (see also the proof of Theorem 1), it appears that the LP-estimator $\widehat{f}_{N}$ solution to the optimization problem (9)-(13) defines the kernel estimator of the support covering all the points $\left(X_{i}, Y_{i}\right)$ and having the smallest surface. Moreover, constraints (11)(13) impose the first derivative $\widehat{f}_{N}^{\prime}$ of the estimator to be Lipschitzian with a particular Lipschitz constant $L_{\widehat{f}_{N}^{\prime}}$ given in Lemma 4 . The constraint (10) says that, for any $X_{i}$, the local linear estimate function $\widehat{f}_{N}\left(X_{i}\right)+\left(x-X_{i}\right) \widehat{f}_{N}^{\prime}\left(X_{i}\right)$ covers all points $\left(X_{j}, Y_{j}\right)$ with $x=X_{j}$ from interval $\left\{x:\left|x-X_{i}\right| \leq h\right\}$. Additionally, the constraints $\alpha_{i} \geq 0$ for all $i=\overline{1, N}$ ensure that $\widehat{f}_{N}(x) \geq 0$ for all $x \in[0,1]$ since the density $K$ is non-negative; this is consistent with the condition of positivity of the estimated boundary function $f(\cdot)$. The constraints $(10)-(11)$ allow you to control the local properties of smoothness estimation $\widehat{f}_{N}$ on the interval $[0,1]$, which are used in the proofs. It is interesting to note that the above described estimator (4), (9)-(13) may be treated as the approximation to Maximum Likelihood Estimate related to the estimation family (4); see BOUCHARD et al. [2] for similar remarks.

\section{Main results}

In the following theorem, the consistency and the convergence rate of the estimator towards the true frontier is established with respect to the $L_{1}$ norm over interval $[0,1]$.

Theorem 1 Let the above mentioned assumptions A1, A2, and B1-B3 hold true and the estimator parameter $C_{\alpha} \geq 8 f_{\max }$. Moreover, let $h \rightarrow 0$ as $N \rightarrow \infty$ such that

$$
\rho_{-}<\liminf _{N \rightarrow \infty} \frac{\log N}{N h^{1+\beta}}, \quad \limsup _{N \rightarrow \infty} \frac{\log N}{N h^{1+\beta}} \leq \rho^{+}<+\infty,
$$

where

$$
\rho_{-}>\frac{f_{\max }}{L_{\beta}} \frac{C_{X} K_{\max }^{\prime}}{10 \times 3^{\beta} K_{\max }^{\prime}+3 C_{\beta}\left(K^{\prime}\right)} \text { and } C_{X}>4 \frac{C_{f}}{f_{\min }} \text {. }
$$


Then, the LP-estimator (4), (9)-(13) with kernel (6) for $(x, t) \in[-h, 1+h]^{2}$ has the following a.s.-properties:

$$
\left\|\widehat{f}_{N}-f\right\|_{1} \leq\left(C_{12}(\beta)\left[\rho_{-}\right]^{-\frac{\beta}{1+\beta}}+2 C_{4}(\beta)\left[\rho^{+}\right]^{\frac{2}{1+\beta}}\right)\left(\frac{\log N}{N}\right)^{\frac{\beta}{1+\beta}}(1+o(1))
$$

asymptotically as $N \rightarrow \infty$ with constants

$$
C_{12}(\beta) \triangleq 5 f_{\max } C_{X} \rho^{+} K_{\max }+10 \times 3^{\beta} L_{\beta} K_{\max }+3 L_{\beta} C_{\beta}(K)
$$

and

$$
C_{4}(\beta) \triangleq 2 L_{\beta}\left(\frac{2 C_{f}}{f_{\min } L_{\beta}}\right)^{\frac{\beta}{1+\beta}}\left(\frac{1}{\rho_{-}}\right)^{\frac{2}{1+\beta}}+7 f_{\max } C_{X} K_{\max }^{\prime}\left(\frac{2 C_{f}}{f_{\min } L_{\beta}}\right)^{\frac{1}{1+\beta}}
$$

Let us highlight that (26) shows that $\widehat{f}_{N}$ reaches (up to a logarithmic factor) the minimax $L_{1}$ rate for frontiers $f$ with Hölder continuous derivative, see Korostelev \& Tsybakov [24], Theorem 4.1.1.

\section{Conclusions}

The results obtained above straightly extend the approach, developed in [2], [3], and [12] under condition $0<\beta \leq 1$, onto more smooth (and periodic) boundary functions when the first derivative function is Hölder continuous with exponent $\beta-1$, and $1<\beta \leq 2$. The estimation method itself for a boundary function, like there in [2], [12], reduces to a linear combination of sufficiently smooth kernel functions, being centered at the sample points, while weighting coefficients are defined by solving a linear programming (LP) problem having minimized a sum of weighting coefficients under related constraints. Note that the related LP problem changes in accordance with the value of $\beta$ in a sense that the constraints composition in LP problem depends on degree of smoothness of boundary function: when $1<\beta \leq 2$ additional inequalities (10) under $X_{i} \neq X_{j}$ include into constraints and the upper bound of second derivative of LP-estimate (11) for all the sample points $X_{i}$. Remind that under $0<\beta \leq 1$ the LP problem of [12] contains the constraints (10) of this work only for $i=j$, i.e., inequalities of type $\widehat{f}_{N}\left(X_{i}\right) \geq Y_{i}$; in addition, the upper bound on first derivative of LP-estimate (11) is used for all the sample points $X_{i}$. In particular it is evident that the transition of the parameter $\beta$ from interval $(0,1]$ to interval $(1,2]$ the number of constraints of LP problem defining LP-estimate increases stepwise which may be considered as a certain fee for providing an optimal estimate under a smoother boundary function. Note as well that all the values of $\beta \in(0,2]$ give the error of estimation in $L_{1}$-norm of the type $O\left((\log N / N)^{\beta /(1+\beta)}\right)$ a.s. under the bandwidth selection of kernel function of type $h \sim(\log N / N)^{1 /(1+\beta)}$. Finally, the authors hope to present an adaptive analog of the proposed method at the given paper for the case of unknown a priori parameter smooth $\beta$. As a conclusion, the authors express their sincere thanks to the anonymous referees for their critical comments contained the stimulating remarks and propositions. 


\section{Appendix}

The proof of Theorem 1 which is given in Subsection 6.4 is based on both upper and lower bounds derived in Subsection 6.2 and Subsection 6.3 respectively. When proving these bounds, we assume that the sequence of the sample $X$-points $\left(X_{i}\right)_{i=\overline{1, N}}$ is already increase ordered, without changing notation from $X_{i}$ to $X_{(i)}$ for the sake of simplicity, that is

$$
X_{i} \leq X_{i+1}, \quad \forall i
$$

We essentially apply the uniform asymptotic bound $O(\log N / N)$ on $\Delta X_{i} \triangleq X_{i}-X_{i-1}$ proved in auxiliary Lemma 7 (see also Lemma A.2 in [12]). Before that, we prove in Subsection 6.1 some preliminary results.

\subsection{Proof of preliminary results}

Proof of Lemma 1. Under assumptions of the Lemma, one may easily demonstrate that the kernel definition (5) ensures the equality (14). Indeed, the LHS (14) may be written from (5) for any $x \in[0,1]$ as follows:

$$
\begin{aligned}
\int_{0}^{1} f(u) K_{h}(x, u) d u & =\int_{0}^{1} f(u) \frac{1}{h} K\left(\frac{x-u}{h}\right) d u \\
& +\int_{0}^{h} f(u) \frac{1}{h} K\left(\frac{x-u-1}{h}\right) d u \\
& +\int_{1-h}^{1} f(u) \frac{1}{h} K\left(\frac{x-u+1}{h}\right) d u
\end{aligned}
$$

and, by changing the variables in the integrals (30)-(32), using the 1-periodicity assumption and the compact support assumption B1, one may obtain

$$
\begin{aligned}
\int_{0}^{1} f(u) K_{h}(x, u) d u & =\int_{(x-1) / h}^{x / h} f(x-h v) K(v) d v \\
& +\int_{(x-1-h) / h}^{(x-1) / h} f(x-h v) K(v) d v \\
& +\int_{x / h}^{(x+h) / h} f(x-h v) K(v) d v \\
& =\int_{-1}^{1} f(x-h v) K(v) d v
\end{aligned}
$$

Thus, (14) is proved. Now (15) follows directly from (14) and assumption B2 for $f(x) \equiv 1$. One obtains from similar arguments to (30)-(36) that

$$
\begin{aligned}
\int_{0}^{1}(x-u) K_{h}(x, u) d u & =h \int_{-1}^{1} v K(v) d v \\
& +\int_{(x-1-h) / h}^{(x-1) / h} K(v) d v \\
& -\int_{x / h}^{(x+h) / h} K(v) d v .
\end{aligned}
$$


Hence, condition $h \leq x \leq 1-h$ implies (16). Finally, equalities (17) and (18) follow directly from the kernel definition (5) and from the assumption B2: for all $u \in[0,1]$ one may easily verify that

$$
\int_{0}^{1} K_{h}(x, u) d x=\int_{-1}^{1} K(v) d v=1
$$

and

$$
\int_{0}^{1}(x-u) K_{h}(x, u) d x=h \int_{-1}^{1} v K(v) d v=0
$$

Proof of Lemma 2 is a straightforward consequence of (5) and assumptions B1 and B2, since

$$
\int_{0}^{1} K_{h}\left(x, X_{i}\right) d x=1 \quad \forall i=\overline{1, N} .
$$

Proof of Lemma 3. For any $x \in[0,1]$, one may write

$$
\begin{aligned}
\left|\widehat{f}_{N}^{\prime}(x)\right| & \leq \sum_{i=1}^{N} \alpha_{i}\left|\frac{d}{d x} K_{h}\left(x, X_{i}\right)\right| \\
& \leq \sup _{u, v}\left|\frac{\partial}{\partial v} K_{h}(v, u)\right| \cdot \sum_{i=1}^{N} \alpha_{i} \mathbf{1}\left\{\left|x-X_{i}\right| \leq h\right\} \\
& \leq 3 K_{\max }^{\prime} C_{\alpha} h^{-1},
\end{aligned}
$$

where constraints (12) are used and give (45). This proves the Lemma.

Proof of Lemma 4. We are to prove (23). Remind that we assume (29) which imply $X_{-}=X_{0}$ and $X_{+}=X_{N+1}$ due to (20). Consider the additional assumption (which holds true for all sufficiently large $N$ ) that is

$$
C_{X}^{2} \frac{\log N}{N h} \leq 5 \frac{L_{\beta} K_{\max }^{\prime \prime}}{C_{\alpha} K_{\max }^{\prime \prime \prime \prime}}
$$

By applying (11) and auxiliary Lemma 7 and Lemma 9 we arrive at

$$
\begin{aligned}
& \max _{x \in\left[X_{0}, X_{N+1}\right]}\left|\widehat{f}_{N}^{\prime \prime}(x)\right| \\
= & \max _{1 \leq i \leq N+1} \max _{x \in\left[X_{i-1}, X_{i}\right]}\left|\widehat{f}_{N}^{\prime \prime}(x)\right| \\
\leq & 2 L_{\beta} K_{\max }^{\prime \prime} \frac{\log N}{N h^{3}}+\frac{1}{8} \max _{1 \leq i \leq N+1}\left[\left(X_{i}-X_{i-1}\right)^{2} \max _{x \in\left[X_{i-1}, X_{i}\right]}\left|\widehat{f}_{N}^{\prime \prime \prime \prime}(x)\right|\right] \\
\leq & 2 L_{\beta} K_{\max }^{\prime \prime} \frac{\log N}{N h^{3}}+\frac{1}{8}\left(C_{X} \frac{\log N}{N}\right)^{2} \max _{x \in\left[X_{0}, X_{N+1}\right]}\left|\widehat{f}_{N}^{\prime \prime \prime \prime}(x)\right|,
\end{aligned}
$$


with $C_{X}>4 C_{f} / f_{\min }$. The maximum term in (50) is bounded as follows: for any $x \in$ $\left[X_{0}, X_{N+1}\right]$,

$$
\begin{aligned}
\left|\widehat{f}_{N}^{\prime \prime \prime \prime}(x)\right| & \leq \sum_{i=1}^{N} \alpha_{i}\left|\frac{d^{4}}{d x^{4}} K_{h}\left(x, X_{i}\right)\right| \\
& \leq \sup _{u, v}\left|\frac{\partial^{4}}{\partial v^{4}} K_{h}(v, u)\right| \cdot \sum_{i=1}^{N} \alpha_{i} \mathbf{1}\left\{\left|x-X_{i}\right| \leq h\right\} \\
& \leq 3 K_{\max }^{\prime \prime \prime \prime} C_{\alpha} h^{-4},
\end{aligned}
$$

since

$$
\sup _{u, v}\left|\frac{\partial^{4}}{\partial v^{4}} K_{h}(v, u)\right| \leq K_{\max }^{\prime \prime \prime \prime} h^{-5} .
$$

Substituting (51) and (53) into (50) and using (46) yield

$$
\begin{aligned}
\max _{x \in\left[X_{0}, X_{N+1}\right]}\left|\widehat{f}_{N}^{\prime \prime}(x)\right| & \leq 2 L_{\beta} K_{\max }^{\prime \prime} \frac{\log N}{N h^{3}}+\frac{3}{8} K_{\max }^{\prime \prime \prime \prime} C_{\alpha}\left(C_{X} \frac{\log N}{N h^{3}}\right)^{2} h^{2} \\
& \leq 4 L_{\beta} K_{\max }^{\prime \prime} \frac{\log N}{N h^{3}} .
\end{aligned}
$$

The result follows.

\subsection{Upper bound for $\widehat{f}_{N}$ in terms of $J_{P}^{*}$}

Lemma 5 Let the assumptions of Theorem 1 hold true. Then for any

$$
\gamma>\left(1+\frac{1}{\beta}\right) L_{\beta} C_{\beta}(K)+f_{\max }\left(5 K_{\max }+K_{\max }^{\prime}\right) C_{X} \rho^{+}
$$

where $C_{X}>4 C_{f} / f_{\min }$ and parameter $\rho^{+}$, meeting (24), and for almost all $\omega \in \Omega$ there exist finite numbers $N_{1}=N_{1}(\omega, \gamma)$ such that for all $N \geq N_{1}$ the LP (9)-(13) is solvable and

$$
J_{P}^{*} \leq C_{f}+\gamma h^{\beta}
$$

Proof of Lemma 5. Recall that $\Delta X_{i}=X_{i}-X_{i-1}>0$ a.s. for all $i$ due to condition (29). Consider arbitrary $N \geq N_{0}(\omega)$ with $N_{0}(\omega)$ from Lemma 7. Introduce function $f_{\gamma}(u)=$ $f(u)+\gamma h^{\beta}$ with parameter $\gamma>0$ and pseudo-estimates

$$
\widetilde{\alpha}_{i}=\sum_{k=-1}^{1} a_{i, k} \int_{X_{i+k-1}}^{X_{i+k}} f_{\gamma}(u) d u, \quad i=\overline{1, N}
$$

where

$$
a_{i, k}=f_{\gamma}\left(X_{i+k}\right)\left(\int_{X_{i+k-1}}^{X_{i+k}} f_{\gamma}(u) d u\right)^{-1} \int_{X_{i+k-1}}^{X_{i+k}} b_{i,-k}(u) d u
$$


and functions $b_{i, k}(\cdot), k=-1,0,1$, represent the coefficients of the 2nd order Lagrange interpolation polynomial for the interval defined by three successive points $X_{i-k-1}<X_{i-k}<$ $X_{i-k+1}$, i.e.,

$$
b_{i, k}(u)=\frac{\prod_{j=-1, j \neq k}^{j=1}\left(u-X_{i-k+j}\right)}{\prod_{j=-1, j \neq k}^{j=1}\left(X_{i}-X_{i-k+j}\right)} .
$$

For any 3 times continuously differentiable function $g:[0,1] \rightarrow \mathbb{R}$ and for all $u \in\left[X_{i-1}, X_{i+1}\right]$, the interpolation error is bounded by

$$
\begin{aligned}
\left|\sum_{k=-1}^{1} b_{i+k, k}(u) g\left(X_{i+k}\right)-g(u)\right| & \leq \max _{u \in\left[X_{i-1}, X_{i+1}\right]}\left|\frac{g^{\prime \prime \prime}(u)}{6} \prod_{j=-1}^{1}\left[u-X_{i+j}\right]\right| \\
& \leq \frac{1}{9 \sqrt{3}}\left[\max _{1 \leq i \leq N+1} \Delta X_{i}\right]_{u \in[0,1]}^{3}\left|g^{\prime \prime \prime}(u)\right| .
\end{aligned}
$$

1. First, we prove constraints (10) under $\alpha_{i}=\widetilde{\alpha}_{i}, i=\overline{1, N}$. For arbitrary $x \in[0,1]$,

$$
\begin{aligned}
& \widetilde{f}_{N}(x) \triangleq \sum_{i=1}^{N} \widetilde{\alpha}_{i} K_{h}\left(x, X_{i}\right)=\sum_{k=-1}^{1} \sum_{i=1}^{N} a_{i, k} \int_{X_{i+k-1}}^{X_{i+k}} f_{\gamma}(u) d u K_{h}\left(x, X_{i}\right) \\
& =\sum_{k=-1}^{1} \sum_{i=1+k}^{N+k} a_{i-k, k} \int_{X_{i-1}}^{X_{i}} f_{\gamma}(u) d u K_{h}\left(x, X_{i-k}\right) \\
& =\sum_{i=1}^{N} \int_{X_{i-1}}^{X_{i}} f_{\gamma}(u) d u\left[a_{i, 0} K_{h}\left(x, X_{i}\right)+a_{i+1,-1} K_{h}\left(x, X_{i+1}\right)\right. \\
& \left.+a_{i-1,1} K_{h}\left(x, X_{i-1}\right)\right] \\
& +a_{1,-1} K_{h}\left(x, X_{1}\right) \int_{X_{-1}}^{X_{0}} f_{\gamma}(u) d u-a_{0,1} K_{h}\left(x, X_{0}\right) \int_{X_{0}}^{X_{1}} f_{\gamma}(u) d u \\
& +a_{N, 1} K_{h}\left(x, X_{N}\right) \int_{X_{N}}^{X_{N+1}} f_{\gamma}(u) d u-a_{N+1,-1} K_{h}\left(x, X_{N+1}\right) \int_{X_{N-1}}^{X_{N}} f_{\gamma}(u) \\
& =\int_{X_{0}}^{X_{N}} f_{\gamma}(u) K_{h}(x, u) d u \\
& +\sum_{i=1}^{N} \int_{X_{i-1}}^{X_{i}} f_{\gamma}(u)\left(\sum_{k=-1}^{1} a_{i+k,-k} K_{h}\left(x, X_{i+k}\right)-K_{h}(x, u)\right) d u,
\end{aligned}
$$

since the sum of the terms (68)-(69) equals zero due to the 1-periodicity of function $f$ and the definition (5) of the kernel $K_{h}$. Particularly, one can verify that $a_{1,-1}=a_{N+1,-1}$, 
$a_{0,1}=a_{N, 1}, K_{h}\left(x, X_{1}\right) \equiv K_{h}\left(x, X_{N+1}\right)$, and $K_{h}\left(x, X_{0}\right) \equiv K_{h}\left(x, X_{N}\right)$. Now we separately bound each of the summands (70)-(71) from below.

Due to the kernel definition (5) and the conditions A1, B1, and B3, the main term (70) is transformed and bounded as follows:

$$
\begin{aligned}
\int_{X_{0}}^{X_{N}} f_{\gamma}(u) K_{h}(x, u) d u= & \int_{0}^{1} f_{\gamma}(u) K_{h}(x, u) d u \\
& +\int_{X_{0}}^{0} f_{\gamma}(u) K_{h}(x, u) d u-\int_{X_{N}}^{1} f_{\gamma}(u) K_{h}(x, u) d u \\
= & \int_{0}^{1} f(u) K_{h}(x, u) d u+\gamma h^{\beta}
\end{aligned}
$$

since the difference of two integrals in (73) vanishes due to periodicity assumption A1 and due to (20) and (6). We continue the integral in (74) by Lemma 1 as follows:

$$
\begin{aligned}
\int_{0}^{1} f_{\gamma}(u) K_{h}(x, u) d u= & \int_{-1}^{1} f(x-h t) K(t) d t+\gamma h^{\beta} \\
= & f(x)+\gamma h^{\beta} \\
& +\int_{-1}^{1}\left[f(x-h t)-f(x)-f^{\prime}(x)(-h t)\right] K(t) d t \\
\geq & f(x)+\left[\gamma-\frac{L_{\beta}}{\beta} C_{\beta}(K)\right] h^{\beta} .
\end{aligned}
$$

Notice that Lemma 7 as well as the definitions for $C_{\beta}(\cdot)$ and $C_{X}$ have been used in (77)-(78).

The $i$-th summand from (71) which is denoted below by $(71)_{i}$ is decomposed and then bounded basing on the 2nd order Lagrange interpolation with the error upper bound (62)- 
(63) being applied for $g(u)=K_{h}(x, u)$ as follows:

$$
\begin{aligned}
(71)_{i} \triangleq & \int_{X_{i-1}}^{X_{i}} f_{\gamma}(u)\left(\sum_{k=-1}^{1} a_{i+k,-k} K_{h}\left(x, X_{i+k}\right)-K_{h}(x, u)\right) d u \\
& \quad \text { by applying definition }(60)] \\
= & f_{\gamma}\left(X_{i}\right) \sum_{k=-1}^{1} \int_{X_{i-1}}^{X_{i}} b_{i+k, k}(u) K_{h}\left(x, X_{i+k}\right) d u-\int_{X_{i-1}}^{X_{i}} f_{\gamma}(u) K_{h}(x, u) d u \\
= & f_{\gamma}\left(X_{i}\right) \int_{X_{i-1}}^{X_{i}}\left(\sum_{k=-1}^{1} b_{i+k, k}(u) K_{h}\left(x, X_{i+k}\right)-K_{h}(x, u)\right) d u \\
& -\int_{X_{i-1}}^{X_{i}}\left(f_{\gamma}(u)-f_{\gamma}\left(X_{i}\right)\right) K_{h}(x, u) d u
\end{aligned}
$$

So, we apply Lemma 7 and the upper bound on the interpolation in (62)-(63):

$$
\begin{aligned}
(71)_{i} \geq & -2 f_{\max } \frac{\left(\max \Delta X_{i}\right)^{3}}{9 \sqrt{3}} \max _{u \in[0,1]}\left|\frac{\partial^{3} K_{h}(x, u)}{\partial u^{3}}\right| \Delta X_{i} \mathbf{1}\left\{\left|x-X_{i}\right| \leq 2 h\right\} \\
& -L_{f}\left(\Delta X_{i}\right) \int_{X_{i-1}}^{X_{i}} K_{h}(x, u) d u \\
\geq & -\left(C_{X} \frac{\log N}{N}\right)^{3} \frac{2 f_{\max } L_{K^{\prime \prime}}}{9 \sqrt{3} h^{4}} \Delta X_{i} \mathbf{1}\left\{\left|x-X_{i}\right| \leq 2 h\right\} \\
& -L_{f} C_{X} \frac{\log N}{N} \int_{X_{i-1}}^{X_{i}} K_{h}(x, u) d u .
\end{aligned}
$$

Moreover, from Lemma 7, it follows that

$$
\sum_{i=1}^{N} \Delta X_{i} \mathbf{1}\left\{\left|x-X_{i}\right| \leq 2 h\right\} \leq 4 h+\frac{C_{X} \log N}{N}
$$

Summing up by $i=\overline{1, N}$ we arrive at the bound for the sum (71) as follows:

$$
\begin{aligned}
{[(71)] } & =\sum_{i=1}^{N}\left[(71)_{i}\right] \\
& \geq-\left[C_{X} \frac{\log N}{N}\right]^{3} \frac{f_{\max } L_{K^{\prime \prime}}}{4.5 \sqrt{3} h^{4}}\left[4 h+\frac{C_{X} \log N}{N}\right]-L_{f} C_{X} \frac{\log N}{N} .
\end{aligned}
$$

Thus, from (64), (72)-(78), (79)-(88), and (70)-(71) it follows for each $j=\overline{1, N}$ that

$$
\widetilde{f}_{N}\left(X_{j}\right) \geq f\left(X_{j}\right)+\delta_{0, N}
$$


with

$$
\begin{aligned}
\delta_{0, N} \triangleq & \left(\gamma-\frac{L_{\beta}}{\beta} C_{\beta}(K)\right) h^{\beta} \\
& -\left(C_{X} \frac{\log N}{N h}\right)^{3} \frac{f_{\max } L_{K^{\prime \prime}}}{\sqrt{3}}-L_{f} C_{X} \frac{\log N}{N}>0
\end{aligned}
$$

for sufficiently large $N \geq N_{0}(\omega)$ when the following inequality holds true:

$$
\gamma-\frac{L_{\beta}}{\beta} C_{\beta}(K) \geq C_{X} \frac{\log N}{N h^{1+\beta}}\left(\left(C_{X} \frac{\log N}{N h}\right)^{2} \frac{f_{\max } L_{K^{\prime \prime}}}{\sqrt{3}}+L_{f} h\right) .
$$

$1^{\prime}$. Similarly, for arbitrary $x \in[0,1]$, we now have to estimate the derivative value

$$
\tilde{f}_{N}^{\prime}(x)=\sum_{i=1}^{N} \widetilde{\alpha}_{i} \frac{d}{d x} K_{h}\left(x, X_{i}\right)=\sum_{i=1}^{N} \widetilde{\alpha}_{i} \widetilde{K}_{h}\left(x, X_{i}\right),
$$

similarly to the arguments (64)-(88). Here

$$
\widetilde{K}_{h}(x, u) \triangleq \frac{\partial}{\partial x} K_{h}(x, u)
$$

with the following upper bound (see (5))

$$
\left|\widetilde{K}_{h}(x, u)\right| \leq h^{-2} \max _{x}\left|K^{\prime}\left(\frac{x-u}{h}\right)\right|=h^{-2} K_{\max }^{\prime} .
$$

Hence, one may repeat the arguments of (65)-(71) by changing $K_{h}$ for $\widetilde{K}_{h}$, and, in particular, equations (64), (70)-(71) give

$$
\begin{aligned}
\widetilde{f}_{N}^{\prime}(x)= & \int_{X_{0}}^{X_{N}} f_{\gamma}(u) \widetilde{K}_{h}(x, u) d u \\
& +\sum_{i=1}^{N} \int_{X_{i-1}}^{X_{i}} f_{\gamma}(u)\left(\sum_{k=-1}^{1} a_{i+k,-k} \widetilde{K}_{h}\left(x, X_{i+k}\right)-\widetilde{K}_{h}(x, u)\right) d u .
\end{aligned}
$$

Therefore, all the rates from (83)-(91) should be divided by $h$, while the value of the main term of decomposition, due to conditions A1, B1-B3, as well as kernel representation (6) is expressed as follows:

$$
\begin{aligned}
\int_{X_{0}}^{X_{N}} f_{\gamma}(u) \widetilde{K}_{h}(x, u) d u & =\frac{1}{h} \int_{-1}^{1} f(x-h t) K^{\prime}(t) d t \\
& =f^{\prime}(x)+\int_{-1}^{1}\left[f^{\prime}(x-h t)-f^{\prime}(x)\right] K(t) d t \\
& =f^{\prime}(x)+\delta_{1, N}
\end{aligned}
$$


with

$$
\left|\delta_{1, N}\right| \leq L_{\beta} C_{\beta}(K) h^{\beta-1}
$$

cf. (72)-(78). Furthermore, the summation of integrals in (97) gives the bound

$$
|(97)| \leq\left(C_{X} \frac{\log N}{N}\right)^{3} \frac{2 f_{\max } L_{K^{\prime \prime \prime}}}{9 \sqrt{3} h^{4}}\left(4+\frac{C_{X} \log N}{N h}\right)+L_{f} C_{X} \frac{\log N}{N h}
$$

instead of (79)-(88). Thus, by taking (101) into account, for sufficiently large $N \geq N_{0}(\omega)$ and for each $x \in[0,1]$ we arrive at

$$
\left|\tilde{f}_{N}^{\prime}(x)-f^{\prime}(x)\right| \leq\left|\delta_{1, N}\right|+O\left(\frac{\log ^{3} N}{N^{3} h^{4}}\right)+O\left(\frac{\log N}{N h}\right) .
$$

$1^{\prime \prime}$. So, we now take (89)-(90) and (103) into account in order to prove constraints (10): for any $\left|X_{j}-X_{i}\right| \leq h$, this yields

$$
\begin{aligned}
\widehat{f}_{N}\left(X_{i}\right)+\left(X_{j}-X_{i}\right) \widehat{f}_{N}^{\prime}\left(X_{i}\right) \geq & f\left(X_{i}\right)+\left(X_{j}-X_{i}\right) f^{\prime}\left(X_{i}\right) \\
& +\delta_{0, N}-h\left|\delta_{2, N}\right| \\
& +O\left(\frac{\log ^{3} N}{N^{3} h^{3}}\right)+O\left(\frac{\log N}{N}\right) \\
\geq & Y_{j}+\delta_{3, N}
\end{aligned}
$$

where (recalling that $\delta_{0, N}$ is defined in $(90)$ )

$$
\begin{aligned}
\delta_{3, N} \triangleq & \delta_{0, N}-L_{\beta} C_{\beta}(K) h^{\beta}+O\left(\frac{\log ^{3} N}{N^{3} h^{3}}\right)+O\left(\frac{\log N}{N}\right) \\
= & \left(\gamma-\left(1+\frac{1}{\beta}\right) L_{\beta} C_{\beta}(K)\right) h^{\beta} \\
& +O\left(\frac{\log ^{3} N}{N^{3} h^{3}}\right)+O\left(\frac{\log N}{N}\right)
\end{aligned}
$$

being positive for sufficiently large $N \geq N_{0}(\omega)$ when both inequalities (92) hold true and, additionally,

$$
\gamma>\left(1+\frac{1}{\beta}\right) L_{\beta} C_{\beta}(K) .
$$

Notice, that inequality (104) implies (92).

$2^{\prime \prime}$. Similarly, constraints (11) hold true under $\alpha_{i}=\widetilde{\alpha}_{i}, i=\overline{1, N}$. Indeed, for arbitrary $x \in[0,1]$, we now have to bound the absolute value of

$$
\widetilde{f}_{N}^{\prime \prime}(x)=\sum_{i=1}^{N} \widetilde{\alpha}_{i} \frac{d^{2}}{d x^{2}} K_{h}\left(x, X_{i}\right)=\sum_{i=1}^{N} \widetilde{\alpha}_{i} \widetilde{\widetilde{K}}_{h}\left(x, X_{i}\right)
$$


instead of (64). Here

$$
\widetilde{\widetilde{K}}_{h}(x, u) \triangleq \frac{\partial^{2}}{\partial x^{2}} K_{h}(x, u)
$$

with the following upper bound (see (5))

$$
\left|\widetilde{\widetilde{K}}_{h}(x, u)\right| \leq h^{-3} \max _{x}\left|K^{\prime \prime}\left(\frac{x-u}{h}\right)\right|=h^{-3} K_{\max }^{\prime \prime} .
$$

Hence, one may repeat the arguments of $(65)-(71)$ by changing $K_{h}$ for $\widetilde{K}_{h}$. Therefore, all the rates from (83)-(90) should be divided by $h^{2}$, while the absolute value of the main term of decomposition, due to conditions B1-B3, is bounded as follows:

$$
\begin{aligned}
& \left|\int_{X_{0}}^{X_{N}} f_{\gamma}(u) \widetilde{\widetilde{K}}_{h}(x, u) d u\right|=\frac{1}{h^{2}}\left|\int_{-1}^{1} f(x-h t) K^{\prime \prime}(t) d t\right| \\
& \leq \frac{1}{h^{2}}\left|\int_{-1}^{1}\left[f(x-h t)-f(x)-f^{\prime}(x)(-h t)\right] K^{\prime \prime}(t) d t\right| \\
& \leq \frac{2 L_{\beta}}{\beta(\beta+1)} K_{\max }^{\prime \prime} h^{\beta-2},
\end{aligned}
$$

instead of (72)-(78). Thus, for sufficiently large $N \geq N_{0}(\omega)$ and for each $X_{j}$ we arrive at

$$
\begin{aligned}
\left|\tilde{f}_{N}^{\prime \prime}\left(X_{j}\right)\right| & \leq \frac{2 L_{\beta} K_{\max }^{\prime \prime}}{\beta(\beta+1) h^{2-\beta}}+O\left(\frac{\log ^{3} N}{N^{3} h^{5}}\right)+O\left(\frac{\log N}{N h^{2}}\right) \\
& \leq \frac{3 L_{\beta}}{\beta \rho_{-}} K_{\max }^{\prime \prime} \frac{\log N}{N h^{3}} .
\end{aligned}
$$

Namely, inequality (112) holds true almost surely for all those $N \geq N_{0}(\omega)$ such that inequalities (118) hold true and

$$
\frac{L_{\beta}}{\beta C_{X}}\left(\frac{1}{\rho_{-}}-\frac{h^{1+\beta} N}{\log N}\right) \geq\left(C_{X} \frac{\log N}{N h}\right)^{2} \frac{f_{\max } L_{K^{\prime \prime \prime \prime}}}{\sqrt{3} K_{\max }^{\prime \prime}}+2 L_{f} h .
$$

3. Finally, the constraints (12) with

$$
C_{\alpha} \geq 4 f_{\max }
$$

also hold true under $\alpha_{i}=\widetilde{\alpha}_{i}, i=\overline{1, N}$. Indeed, by Lemma 7 the following inequalities hold a.s. for all $N \geq N_{0}(\omega)$ and for each $j=\overline{1, m_{h}}$, where $m_{h}=\left\lfloor h^{-1}\right\rfloor$ :

$$
\begin{aligned}
& \sum_{i=1}^{N} \widetilde{\alpha}_{i} \mathbf{1}\left\{(j-1) / m_{h} \leq X_{i}<j / m_{h}\right\} \\
\leq & \left(f_{\max }+\gamma h^{\beta}\right)\left(1 / m_{h}+2 C_{X} \frac{\log N}{N}\right) \\
\leq & 4 f_{\max } h,
\end{aligned}
$$


under additional assumptions

$$
f_{\max } \geq \gamma h^{\beta}, \quad h \geq 2 C_{X} \log N / N .
$$

Thus, constraints (12) are fulfilled under (114) almost surely, for any sufficiently large $N$.

4. Since all $\widetilde{\alpha}_{i} \geq 0$, constraints (13) hold true. Hence, vector $\left(\widetilde{\alpha}_{1}, \ldots, \widetilde{\alpha}_{N}\right)^{T}$ is the admissible point for the LP (9)-(13). Now inequality (58) follows from Lemma 2.

\subsection{Lower bound for estimate $\widehat{f}_{N}$}

Lemma 6 Under the assumptions of Theorem 1, for almost all $\omega \in \Omega$ there exist finite numbers $N_{2}(\omega)$ such that for any $x \in[0,1]$ and for all $N \geq N_{2}(\omega)$

$$
\widehat{f}_{N}(x)-f(x) \geq-\frac{2 L_{\beta}}{\beta} h^{\beta}-\left(\frac{2 C_{f}}{f_{\min }}+2 L_{\beta} K_{\max }^{\prime \prime}+4 C_{\alpha} K_{\max }^{\prime} C_{X}\right) \frac{\log N}{N h}
$$

assuming that $L_{f} h \leq C_{\alpha} K_{\max }^{\prime}$ and $C_{X}>4 C_{f} / f_{\min }$.

Proof of Lemma 6. Let us take use of Lemma 8 and its Corollary 1 introducing

$$
\delta_{y} \sim \frac{\log N}{N h}, \quad \delta_{x} \sim h .
$$

Thus, for any $N \geq N_{6}(\omega)$ and any $x \in[0,1]$ there exist (with probability one) integers $i_{k} \in\{1, \ldots, N\}, k=\overline{1, m_{h}}$, such that

$$
\left|x-X_{i_{k}}\right| \leq \delta_{x}
$$

and inequality (154) that is

$$
\mathcal{L}_{x} f\left(X_{i_{k}}\right) \leq Y_{i_{k}}+\delta_{y}+\frac{L_{\beta}}{2} \delta_{x}^{\beta}
$$

Now, we put a point $x \in[0,1]$, find index $i_{x} \in\{1, \ldots, N\}$ such that $\left|X_{i_{x}}-X_{i_{k}}\right| \leq h$ and

$$
\left|x-X_{i_{x}}\right| \leq \max \left\{\Delta X_{i_{x}-1}, \Delta X_{i_{x}}\right\} \leq C_{X} \frac{\log N}{N}
$$

then the estimation error at point $x$ can be decomposed as

$$
\begin{aligned}
f(x)-\widehat{f}_{N}(x) & =\left[f(x)-f\left(X_{i_{x}}\right)\right] \\
& +\left[f\left(X_{i_{x}}\right)-\mathcal{L}_{X_{i_{x}}} f\left(X_{i_{k}}\right)\right] \\
& +\left[\mathcal{L}_{X_{i_{x}}} f\left(X_{i_{k}}\right)-\mathcal{L}_{X_{i_{x}}} \widehat{f}_{N}\left(X_{i_{k}}\right)\right] \\
& +\left[\mathcal{L}_{X_{i_{x}}} \widehat{f}_{N}\left(X_{i_{k}}\right)-\widehat{f}_{N}\left(X_{i_{k}}\right)\right] \\
& +\left[\widehat{f}_{N}\left(X_{i_{k}}\right)-\widehat{f}_{N}(x)\right] .
\end{aligned}
$$


The first and the last decomposition components, i.e. RHS (123) and (127), can be similarly bounded by using the proper Lipschitz constants as follows:

$$
\begin{aligned}
\left|f(x)-f\left(X_{i_{x}}\right)\right| & \leq L_{f}\left|x-X_{i_{x}}\right| \leq L_{f} C_{X} \frac{\log N}{N} \\
\left|\widehat{f}_{N}\left(X_{i_{k}}\right)-\widehat{f}_{N}(x)\right| & \leq L_{\widehat{f}_{N}}\left|x-X_{i_{x}}\right| \leq L_{\widehat{f}_{N}} C_{X} \frac{\log N}{N} .
\end{aligned}
$$

The similar decomposition components (124) and (126) may be bounded as follows:

$$
\begin{aligned}
\left|f\left(X_{i_{x}}\right)-\mathcal{L}_{X_{i_{x}}} f\left(X_{i_{k}}\right)\right| & \leq \frac{L_{\beta}}{\beta}\left|X_{i_{x}}-X_{i_{k}}\right|^{\beta} \leq \frac{L_{\beta}}{\beta} \delta_{x}^{\beta}, \\
\left|\mathcal{L}_{X_{i_{x}}} \widehat{f}_{N}\left(X_{i_{k}}\right)-\widehat{f}_{N}\left(X_{i_{x}}\right)\right| & \leq \frac{L_{\widehat{f}_{N}^{\prime}}}{2}\left|X_{i_{x}}-X_{i_{k}}\right|^{2} \leq \frac{L_{\widehat{f}_{N}^{\prime}}}{2} \delta_{x}^{2},
\end{aligned}
$$

with Lipschitz constant $L_{\widehat{f}_{N}}$ for the derivative estimator function $\widehat{f}_{N}^{\prime}(x)$. Finally, we bound the central decomposition component (125) by applying Corollary 1 of Lemma 8 with

$$
\delta_{x}=h, \quad \delta_{y}=\frac{2 C_{f}}{f_{\min }} \frac{\log N}{N h},
$$

and using the estimator constraints (10); we obtain

$$
\begin{aligned}
\mathcal{L}_{X_{i_{x}}} f\left(X_{i_{k}}\right)-\mathcal{L}_{X_{i_{x}}} \widehat{f}_{N}\left(X_{i_{k}}\right) & \leq Y_{i_{k}}+\delta_{y}+\frac{L_{\beta}}{2} \delta_{x}^{\beta}-Y_{i_{k}} \\
& =\frac{2 C_{f}}{f_{\min }} \frac{\log N}{N h}+\frac{L_{\beta}}{2} h^{\beta}
\end{aligned}
$$

Therefore, equations (123)-(134) and Lemmas 3 and 4 lead to the lower bound

$$
\begin{aligned}
& \widehat{f}_{N}(x)-f(x) \\
\geq & -\left(\frac{2 C_{f}}{f_{\min }} \frac{\log N}{N h}+\frac{2 L_{\beta}}{\beta} h^{\beta}+\frac{L_{\widehat{f}_{N}^{\prime}}^{\prime}}{2} h^{2}+\left(L_{f}+L_{\widehat{f}_{N}}\right) C_{X} \frac{\log N}{N}\right) \\
\geq & -\frac{2 L_{\beta}}{\beta} h^{\beta}-\left(\frac{2 C_{f}}{f_{\min }}+2 L_{\beta} K_{\max }^{\prime \prime}+4 C_{\alpha} K_{\max }^{\prime} C_{X}\right) \frac{\log N}{N h}
\end{aligned}
$$

assuming additionally that

$$
L_{f} h \leq C_{\alpha} K_{\max }^{\prime}
$$

Thus, the obtained lower bound holds true for any sufficiently large $N$ (starting from random a.s. finite integer, which does not depend on $x$ ). Lemma 6 is proved.

Remark 7 The optimal order of the lower bound, proved in Lemma 6, is attained by

$$
h=h_{1}\left(\frac{\log N}{N}\right)^{\frac{1}{1+\beta}}
$$


when two terms in (137) are balanced, and the lower bound in (135)-(137) becomes

$$
\widehat{f}_{N}(x)-f(x) \geq-C_{L B}\left(h_{1}\right)\left(\frac{\log N}{N}\right)^{\frac{\beta}{1+\beta}}
$$

where constant

$$
C_{L B}\left(h_{1}\right)=\frac{2 L_{\beta}}{\beta} h_{1}^{\beta}+\left(\frac{2 C_{f}}{f_{\min }}+2 L_{\beta} K_{\max }^{\prime \prime}+4 C_{\alpha} K_{\max }^{\prime} C_{X}\right) \frac{1}{h_{1}}
$$

may be optimized by $h_{1}>0$. It is interesting to observe that four last components of the estimation error decomposition (123)-(127) become of the same order while the first one RHS (123) be negligible, see (128)-(134).

\subsection{Proof of Theorem 1}

1. Since $|u|=u-2 u \mathbf{1}\{u<0\}$, the $L_{1}$-norm of estimation error can be expanded as

$$
\begin{aligned}
\left\|\widehat{f}_{N}-f\right\|_{1} & =\int_{0}^{1}\left[\widehat{f}_{N}(x)-f(x)\right] d x \\
& +2 \int_{0}^{1}\left[f(x)-\widehat{f}_{N}(x)\right] \mathbf{1}\left\{\widehat{f}_{N}(x)<f(x)\right\} d x .
\end{aligned}
$$

2. Applying Lemmas 2 and 5 to the right hand side (141) yields

$$
\limsup _{N \rightarrow \infty} h^{-\beta}\left(\int_{0}^{1}\left[\widehat{f}_{N}(x)-f(x)\right] d x\right) \leq \gamma \quad \text { a.s. }
$$

where $\gamma>0$ is large enough.

3. In order to obtain a similar result for the term (142), note that Lemma 6 implies

$$
\zeta_{N}(x, \omega) \triangleq \varepsilon_{L B}^{-1}(N)\left[f(x)-\widehat{f}_{N}(x)\right] \leq \text { const }<\infty \quad \text { a.s. }
$$

uniformly with respect to both $x \in[0,1]$ and $N \geq N_{2}(\omega)$, with

$$
\varepsilon_{L B}(N) \triangleq \operatorname{const} \frac{\log N}{N h}
$$

with finite const $>0$. Hence, one may apply Fatou's lemma, taking into account that $u \mathbf{1}\{u>0\}$ is a continuous, monotone function:

$$
\begin{aligned}
& \limsup _{N \rightarrow \infty} \varepsilon_{L B}^{-1}(N) \int_{0}^{1}\left[f(x)-\widehat{f}_{N}(x)\right] \mathbf{1}\left\{\widehat{f}_{N}(x)<f(x)\right\} d x \\
\leq & \int_{0}^{1} \limsup _{N \rightarrow \infty} \zeta_{N}(x, \omega) \mathbf{1}\left\{\zeta_{N}(x, \omega)>0\right\} d x \\
\leq & \text { const }<\infty \text { a.s. }
\end{aligned}
$$

4. Finally, we put bandwidth $h$ from the balancing assumption

$$
h^{\beta} \sim \frac{\log N}{N h} .
$$

Thus, the obtained relations together with (141) and (142) imply (26). Theorem 1 is proved. 
The following results are quoted here for the sake of completeness.

Lemma 7 ((Lemma A.2 in [12])) Let function $f:[0,1] \rightarrow \mathbb{R}$ meets the assumption A1 and sequence $\left(X_{i}\right)_{i=\overline{1, N}}$ be obtained from an independent sample with p.d.f. $f(x) / C_{f}$ by increase ordering (29), where $C_{f}$ is defined by (2). Denote $X_{0}=0$ and $X_{N+1}=1$. Then for any finite constant $C_{X}>4 C_{f} / f_{\min }$ there exist almost surely finite number $N_{0}=N_{0}(\omega)$ such that

$$
\max _{i=\overline{1, N+1}} \Delta X_{i} \leq C_{X} \frac{\log N}{N} \quad \forall N \geq N_{0}
$$

with probability 1. For instance, one may fix constant $C_{X}$ as follows:

$$
C_{X}=5 f_{\max } / f_{\min }
$$

Lemma 8 ((Lemma A.3 in [12])) Let random sample $\left\{\left(X_{i}, Y_{i}\right) \mid i=\overline{1, N}\right\}$ be defined as in Section 2. Let sequence $\delta_{x}=\delta_{x}(N)$ be positive, and for some $\varepsilon>0$

$$
\liminf _{N \rightarrow \infty} N^{1-\varepsilon} \delta_{x}>0 .
$$

Define

$$
m_{\delta} \triangleq \min \left\{\text { integer } m: m \geq \delta_{x}^{-1}\right\}
$$

and assume a positive sequence $\delta_{y}=\delta_{y}(N)<f_{\min }$ meeting for all sufficiently large $N$ the inequality

$$
\delta_{y} \geq \kappa m_{\delta} \frac{\log N}{N}, \quad \text { with } \quad \kappa>\frac{(2-\varepsilon) C_{f}}{f_{\min }} .
$$

Then, under the assumptions of Lemma 7, with probability 1, there exists finite number $N_{6}(\omega)$ such that for any $N \geq N_{6}(\omega)$ there is such a subset of points $\left\{\left(X_{i_{k}}, Y_{i_{k}}\right), k=\overline{1, m_{\delta}}\right\}$ in the sample $\left\{\left(X_{i}, Y_{i}\right), i=\overline{1, N}\right\}$, that the following inequalities hold:

$$
(k-1) / m_{\delta} \leq X_{i_{k}}<k / m_{\delta}, \quad f\left(X_{i_{k}}\right)-\delta_{y} \leq Y_{i_{k}} \leq f\left(X_{i_{k}}\right) .
$$

Corollary 1 Let $\delta_{x}$ and $\delta_{y}$ meet the conditions of Lemma 8. Then, with probability 1, for any $N \geq N_{6}(\omega)$ and any $x \in[0,1]$ there exists integer $i_{k} \in\{1, \ldots, N\}$ such that $\left|x-X_{i_{k}}\right| \leq \delta_{x}$ and $f\left(X_{i_{k}}\right)-\delta_{y} \leq Y_{i_{k}} \leq f\left(X_{i_{k}}\right)$. Furthermore, if constant Lipschitz $L_{\beta}<\infty$ with $1<\beta \leq 2$, then

$$
\mathcal{L}_{x} f\left(X_{i_{k}}\right) \leq Y_{i_{k}}+\delta_{y}+\frac{L_{\beta}}{2} \delta_{x}^{\beta} .
$$

Lemma 9 ((Lemma A.4 in [12])) Let function $g:[0, \Delta] \rightarrow \mathbb{R}$ be twice continuous differentiable, $\Delta>0$. Then

$$
\max _{x \in[0, \Delta]}|g(x)| \leq \max \{|g(0)|,|g(\Delta)|\}+\frac{\Delta^{2}}{8} \max _{x \in[0, \Delta]}\left|g^{\prime \prime}(x)\right| \text {. }
$$




\section{References}

[1] Aragon, Y., Daouia, A., and Thomas-Agnan, C. (2005) Nonparametric frontier estimation: a conditional quantile-based approach. Journal of Econometric Theory, 21(2), $358-389$.

[2] Bouchard, G., Girard, S., Iouditski A.B., and Nazin A.V. (2004) Nonparametric Frontier Estimation by Linear Programming. Automation and Remote Control, 65(1), 5864.

[3] Bouchard, G., Girard, S., Iouditski A., and Nazin A. (2005) Some linear programming methods for frontier estimation. Applied Stochastic Models in Business and Industry, 21(2), 175-185.

[4] Cazals, C., Florens, J.-P., and Simar, L. (2002) Nonparametric frontier estimation: A robust approach. Journal of Econometrics, 106(1), 1-25.

[5] Daouia, A., and Simar, L. (2005) Robust nonparametric estimators of monotone boundaries. Journal of Multivariate Analysis, 96, 311-331.

[6] Deprins, D., Simar, L. and Tulkens, H. (1984) Measuring Labor Efficiency in Post Offices. in The Performance of Public Enterprises: Concepts and Measurements by M. Marchand, P. Pestieau and H. Tulkens, North Holland ed, Amsterdam.

[7] Farrel, M.J. (1957) The measurement of productive efficiency. Journal of the Royal Statistical Society A, 120, 253-281.

[8] Geffroy, J. (1964) Sur un problème d'estimation géométrique. Publications de l'Institut de Statistique de l'Université de Paris, XIII, 191-200.

[9] Gijbels, I., Mammen, E., Park, B.U. and Simar, L. (1999) On estimation of monotone and concave frontier functions. Journal of the American Statistical Association, 94, $220-228$.

[10] Girard, S., Guillou, A., and Stupfler, G. (2013) Frontier estimation with kernel regression on high order moments. Journal of Multivariate Analysis, 116, 172-189.

[11] Girard, S., Guillou, A., and Stupfler, G. (2014) Uniform strong consistency of a frontier estimator using kernel regression on high order moments. ESAIM: Probability and Statistics, to appear.

[12] Girard, S., Iouditski, A.B., and Nazin, A.V. (2005) $L_{1}$-Optimal Nonparametric Frontier Estimation via Linear Programming. Automation and Remote Control, 66(12), 20002018.

[13] Girard, S., and Jacob, P. (2003a) Extreme values and Haar series estimates of point processes boundaries. Scandinavian Journal of Statistics, 30(2), 369-384.

[14] Girard, S., and Jacob, P. (2003b) Projection estimates of point processes boundaries. Journal of Statistical Planning and Inference, 116(1), 1-15. 
[15] Girard, S., and Jacob, P. (2004) Extreme values and kernel estimates of point processes boundaries. ESAIM: Probability and Statistics, 8, 150-168.

[16] Girard, S., and Jacob, P. (2008) Frontier estimation via kernel regression on high power transformed data. Journal of Multivariate Analysis, 99, 403-420.

[17] Girard, S., and Menneteau, L. (2005) Central limit theorems for smoothed extreme values estimates of point processes boundaries. Journal of Statistical Planning and Inference, 135(2), 433-460.

[18] Hall, P., Park, B. U., and Stern, S. E. (1998) On polynomial estimators of frontiers and boundaries. Journal of Multivariate Analysis, 66, 71-98.

[19] Härdle, W., Hall, P., and Simar, L. (1995) Iterated bootstrap with application to frontier models. J. Productivity Anal., 6, 63-76.

[20] Härdle, W., Park, B. U., and Tsybakov, A. B. (1995) Estimation of a non sharp support boundaries. Journal of Multivariate Analysis, 43, 205-218.

[21] Jacob, P., and Suquet, P. (1995) Estimating the edge of a Poisson process by orthogonal series. Journal of Statistical Planning and Inference, 46, 215-234.

[22] Knight, K. (2001) Limiting distributions of linear programming estimators. Extremes, 4(2), 87-103.

[23] Korostelev, A., Simar, L., and Tsybakov, A. B. (1995) Efficient estimation of monotone boundaries. The Annals of Statistics, 23, 476-489.

[24] Korostelev, A.P., and Tsybakov, A.B. (1993) Minimax theory of image reconstruction. in Lecture Notes in Statistics, 82, Springer-Verlag, New York.

[25] Menneteau, L. (2008) Multidimensional limit theorems for smoothed extreme value estimates of point processes boundaries. ESAIM: Probability and Statistics, 12, 273307.

Nazin A.V., Trapeznikov Institute of Control Sciences RAS, 65, Profsoyuznaya str., Moscow, Russia, nazine@ipu.ru

Girard S., LJK, Inria Grenoble Rhône-Alpes, équipe-projet Mistis, Inovallée, 655, av. de l'Europe, Montbonnot, 38334 Saint-Ismier cedex, France, stephane.girard@inria.fr 